\title{
Overview of Early Cases of Coronavirus Disease 2019 (COVID-19) at a Tertiary Care Centre in North India
}

Shrikant $\underline{\text { Sharma }},{ }^{1} M D$, Prakash $\underline{\text { Keswani }},{ }^{1} M D, D N B$ (Endocrinol), Abhishek Bhargava, ${ }^{1}{ }_{M B B S}$, Ramji Sharma, ${ }^{1} M D$, Ajeet Shekhawat, ${ }^{1} M D$, Sudhir Bhandari, ${ }^{1}{ }_{M D}$

\begin{abstract}
Introduction: As the coronavirus disease 2019 (COVID-19) pandemic continues to spread on an unprecedented scale from around the world, we described our experience in treating early COVID-19 cases in India. Materials and Methods: An observational study of COVID-19 patients admitted to a tertiary care centre in North India between 2 March-4 April 2020 was performed. The clinical, epidemiological, laboratory, treatment and outcome data of patients were evaluated. Results: A total of 75 patients were treated and $56(74.66 \%)$ were men. The clinical spectrum of COVID-19 ranged from asymptomatic to acute respiratory distress syndrome (ARDS). Fever (85.36\%) was the most common symptom followed by cough $(56.09 \%)$ and dyspnoea $(\mathbf{1 9 . 5 1 \% )}$. Findings from hemogram analysis showed that $32 \%, 21.33 \%$ and $18.67 \%$ of patients had lymphopaenia, eosinopenia and thrombocytopaenia, respectively. Inflammatory markers such as C-reactive protein, D-dimer, ferritin, fibrin degradation product and interleukin-6 were significantly elevated $(P<0.05)$ in patients who required oxygen therapy than those who did not require it, suggesting the potential role such markers could play in predicting prognosis in patients. Mean hospital stay was 9.2 days and $72(96 \%)$ patients made a complete recovery, but $3(4 \%)$ patients demised after progressing to ARDS. Conclusion: The clinical and epidemiological spectrum of COVID-19 has jeopardised the health system in India. Without a proven therapy to combat this pandemic and with no sight of vaccines in the near future, a preventive strategy should be adopted to contain the spread of this infectious disease.
\end{abstract}

Ann Acad Med Singapore 2020;49:449-55

Key words: Acute respiratory distress syndrome, Cytokine release syndrome, Interleukin-6, Lopinavir-ritonavir, Lymphopaenia

\section{Introduction}

The novel coronavirus disease 2019 (COVID-19) emerged in Wuhan City in Mainland China in December 2019. It belonged to the genus betacoronavirus that was previously linked to severe acute respiratory syndrome coronavirus (SARS-CoV) and Middle East respiratory syndrome coronavirus (MERS-CoV) which caused widespread mortality. ${ }^{1}$ Compared to SARS-CoV and MERS-CoV, COVID-19 spreads at a faster rate and is also more contagious. ${ }^{2}$ Within 3 months, it has spread across $>200$ countries and poses a huge challenge to many health systems from around the world. On 11 March 2020, the World Health Organization declared COVID-19 a pandemic. ${ }^{3}$

The clinical spectrum of COVID-19 ranges from asymptomatic to onset of acute respiratory distress syndrome (ARDS). ${ }^{4,5}$ Disease course is contingent on age, comorbidities and immunological health. It is hypothesised that in a particular subgroup of patients, a cascade of inflammatory reactions may be initiated that result in the devastating cytokine release syndrome. ${ }^{6}$

On 30 January 2020, India reported her first 3 cases of COVID-19 in the state of Kerala. On 2 March 2020, the $4^{\text {th }}$ case - a tourist from Italy — was reported by our centre.

${ }^{1}$ Department of Medicine, SMS Medical College, India

Address for Correspondence: A/Prof Shrikant Sharma, Department of Medicine, SMS Medical College, Vijaybarisikar Road, Plot 47 Path 6, Jaipur 302039, Rajasthan, India.

Email: Skant_sunita@yahoo.co.in 
Over 40 days, Rajasthan reported 570 COVID-19 cases; over the same period, India reported $>7300$ COVID-19 cases and 242 deaths. ${ }^{7}$

At the time of writing, there is no vaccine or specific treatment available for this novel coronavirus. Consequently, it is necessary to identify potential risk factors and observe the clinical course of the disease which can help to predict disease progression and severity in COVID-19 patients. This study described the clinical, epidemiological, laboratory and radiological characteristics, treatment history and outcome of COVID-19 patients admitted to a tertiary care centre in North India.

\section{Materials and Methods}

Patients who were admitted to our centre in North India between 2 March-4 April 2020 and tested positive for COVID-19 on reverse transcriptase-polymerase chain reaction assays - using the viral nucleic acid detection kit recommended by the National Institute of Virology, India-were included in the study. Nasopharyngeal and oropharyngeal swabs were collected from suspected patients into collection tubes that contained virus transport medium for extraction of total ribonucleic acid.

Details on clinical, epidemiological, laboratory and radiological characteristics, treatment and outcomes of patients were retrieved from electronic medical records and history-taking from patients. The data was stratified according to age groups and analysed.

Baseline investigations included blood cell count, liver function and renal function tests, quantitative assessment of lactate dehydrogenase (LDH), C-reactive protein (CRP), D-dimer, fibrin degradation products (FDP), interleukin-6 (IL-6), procalcitonin and serum ferritin. Radiological investigations included chest radiographs and computed tomography (CT) scans (for selected cases). Electrocardiogram (ECG) was performed regularly to monitor changes in ST-segment, T-waves and QT interval.

Data were entered using EpiData version 3.03 (EpiData Association, Odense, Denmark) and data analysis was done using $\mathrm{R}$ version 3.5.2 (The $\mathrm{R}$ Foundation for Statistical Computing, Vienna, Austria). Comparison of lymphopaenia, eosinopenia and thrombocytopaenia in the various age groups was performed with chi-square test. Fisher's Exact test was used to examine inflammatory markers in patients who required oxygen therapy and those who did not require it. Categorical variables were expressed as $95 \%$ confidence interval (CI). A value of $P<0.05$ was considered statistically significant.

\section{Results}

A total of 75 patients with a mean age of 38.46 years who were diagnosed with COVID-19 were included in the study. The mean age of patients who required oxygen therapy was 59.8 years. All patients who required oxygen therapy were men. Mean age of patients who did not require oxygen therapy was 35.91 years. Most patients $(29.3 \%)$ were aged between 19-30 years old and those aged $<18$ years old $(12 \%)$ had the least number of patients. There was a preponderance of male patients $(74.66 \%)$ in all age groups except those aged $<18$ years old where more female $(66.7 \%)$ patients were seen.

Of the 19 (25.3\%) patients who had a history of foreign travel to countries such as Dubai, Italy, Oman, Saudi Arabia and Spain, $14(73.6 \%)$ were men and most of them were aged between 19-45 years old. The remaining patients had contracted COVID-19 locally in areas where there was an outbreak of the disease.

Thirty-four $(45.33 \%)$ patients were asymptomatic (Table 1). In symptomatic patients, fever $(85.36 \%)$ was the most common symptom followed by cough $(56.09 \%)$ and myalgia (46.34\%). Shortness of breath was seen in $8(19.51 \%)$ patients. In patients who required oxygen therapy, 2 were aged between 31-45 years old and 6 were $>61$ years old.

At admission, 8 (10.67\%) patients had abnormal chest radiograph findings. The most common finding was bilateral pneumonitis (Table 1). Common findings on CT scans included multifocal patchy areas of consolidation bilaterally with surrounding ground-glass opacities and interstitial pneumonitis.

Comorbidities were identified in 10 (13.3\%) patients and some patients had multiple underlying comorbidities. Diabetes and hypertension were reported in $3(4 \%)$ and $5(6.6 \%)$ patients, respectively. Two (2.7\%) patients had a history of cardiovascular disease and another $2(2.7 \%)$ had hypothyroidism, while $1(1.3 \%)$ patient each had a history of cerebrovascular accident and chronic obstructive pulmonary disease, respectively. Comorbidities were identified in $6(75 \%)$ patients who required oxygen therapy.

Findings of hemogram analysis showed that lymphopaenia, eosinopenia and thrombocytopaenia were seen in $24(32 \%), 16(21.3 \%)$ and $14(18.7 \%)$ patients, respectively. Lymphopaenia was the most common finding in patients aged $>61$ years old and was seen in $7(58.3 \%)$ patients (Table 2). It was 5.3 (95\% CI 0.8-35.4) times higher in this group of patients than those aged $<18$ years old. However, this 

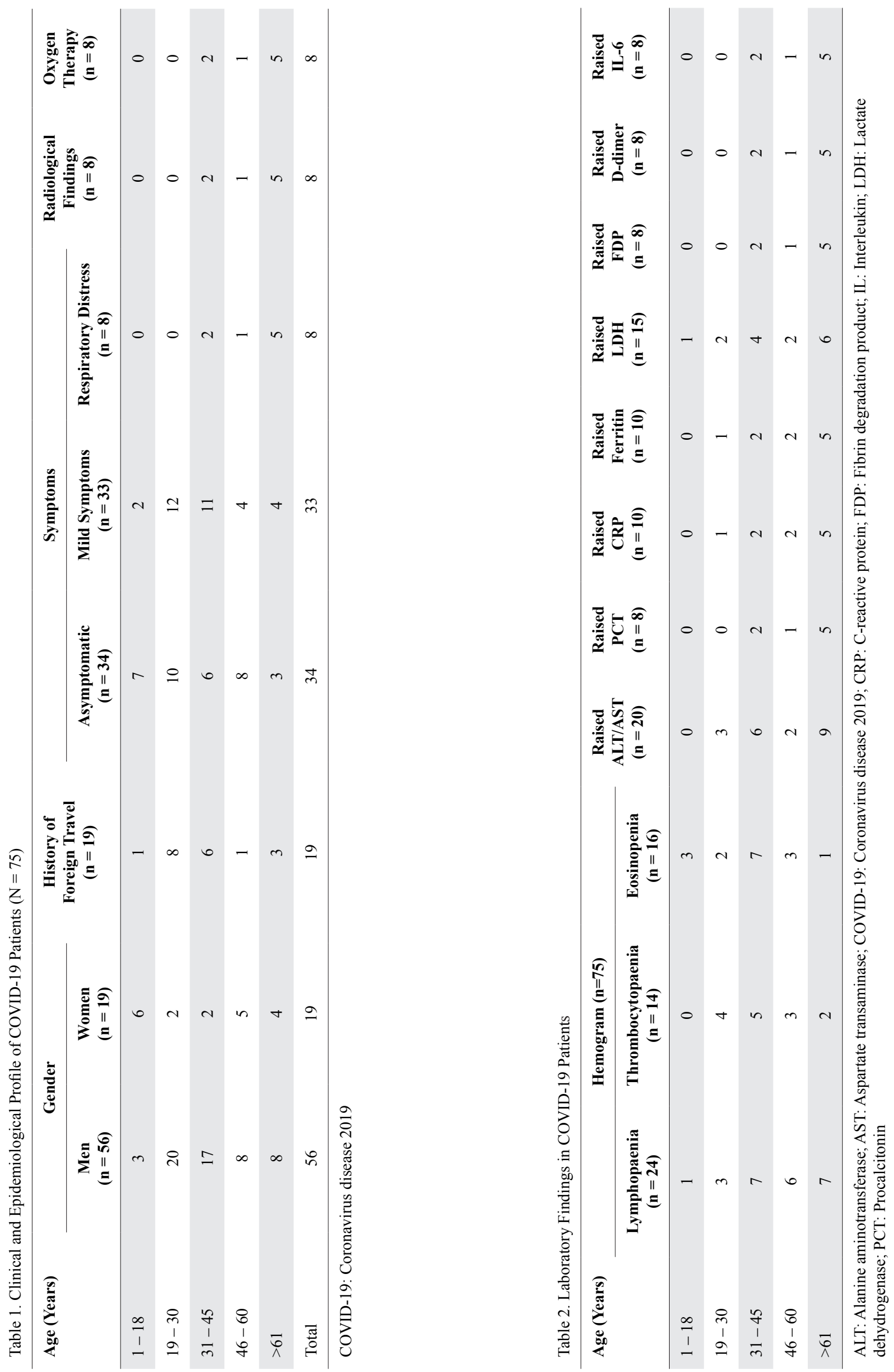
Table 3. Laboratory Parameters of COVID-19 Patients on Oxygen Therapy

\begin{tabular}{|c|c|c|c|c|}
\hline Parameter $^{*}$ & $\begin{array}{l}\text { All Patients } \\
\quad(n=75)\end{array}$ & $\begin{array}{l}\text { No Oxygen Therapy } \\
(n=67)\end{array}$ & $\begin{array}{l}\text { Oxygen Therapy } \\
\qquad(n=8)\end{array}$ & $P$ Value \\
\hline \multicolumn{5}{|l|}{ Blood cell count $(\%)$} \\
\hline $\mathrm{WBC},<1.5 \times 10^{9} / \mathrm{L}$ & $24(32.0)$ & $17(25.3)$ & $7(87.5)$ & $<0.05$ \\
\hline Platelet, $<1.4 \times 10^{9} / \mathrm{L}$ & $14(18.67)$ & $13(19.4)$ & $1(12.5)$ & 0.503 \\
\hline Eosinophils, $<1 \%$ of total WBC & $16(21.33)$ & $15(22.3)$ & $1(12.5)$ & $>0.05$ \\
\hline C-reactive protein $(\mathrm{mg} / \mathrm{dL})$ & $3.5(1.2-9.4)$ & $2.85(1.2-6.0)$ & $7.2(4.2-9.4)$ & $<0.05$ \\
\hline$>5 \mathrm{mg} / \mathrm{L}(\%)$ & $10(13.33)$ & $3(4.47)$ & $7(87.5)$ & \\
\hline Procalcitonin (ng/mL) & $0.223(0.04-1.30)$ & $0.215(0.04-0.70)$ & $0.775(0.40-1.30)$ & $<\mathbf{0 . 0 5}$ \\
\hline$>0.5 \mathrm{ng} / \mathrm{L}(\%)$ & $8(10.67)$ & $1(1.49)$ & $7(87.5)$ & \\
\hline$>460 \mathrm{U} / \mathrm{L}(\%)$ & $15(20.0)$ & $8(11.94)$ & $7(87.5)$ & \\
\hline Aspartate transaminase (U/L) & $34.5(10-122)$ & $29(10-94)$ & $74(59-122)$ & $<0.05$ \\
\hline$>40 \mathrm{U} / \mathrm{L}(\%)$ & $20(26.67)$ & $13(19.40)$ & $7(87.5)$ & \\
\hline Alanine aminotransferase (U/L) & $34.1(15-130)$ & $27(15-64)$ & $74(52-130)$ & $<\mathbf{0 . 0 5}$ \\
\hline$>40 \mathrm{U} / \mathrm{L}(\%)$ & $20(26.67)$ & $13(19.40)$ & $7(87.5)$ & \\
\hline Fibrin degradation product $(\mu \mathrm{g} / \mathrm{mL})$ & $3.2(1.3-9.2)$ & $2.95(1.3-5.5)$ & $6.95(4.2-9.2)$ & $<0.05$ \\
\hline$>5 \mu \mathrm{g} / \mathrm{mL}(\%)$ & $8(10.67)$ & $1(1.49)$ & $7(87.5)$ & \\
\hline D-dimer $(\mu \mathrm{g} / \mathrm{mL})$ & $0.30(0.10-1.9)$ & $0.26(0.10-0.58)$ & $1.45(0.20-1.9)$ & $<0.05$ \\
\hline$>0.5 \mu \mathrm{g} / \mathrm{mL}(\%)$ & $8(10.67)$ & $1(1.49)$ & $7(87.5)$ & \\
\hline Interleukin-6 (pg/mL) & $5.2(2.3-570)$ & $4.92(2.3-7.9)$ & $73.5(12-570)$ & $<0.05$ \\
\hline$>7 \mathrm{pg} / \mathrm{mL}(\%)$ & $8(10.67)$ & $0(0.0)$ & $8(100.0)$ & \\
\hline
\end{tabular}

COVID-19: Coronavirus disease 2019; WBC: White blood cell

*Values are in median (interquartile range) unless otherwise stated.

difference was not statistically significant. Eosinopenia and thrombocytopaenia were the most common findings in patients aged between 31-45 years old and were seen in $7(36.8 \%)$ and $5(26.3 \%)$ patients, respectively.

CRP and serum ferritin were elevated in $10(13.3 \%)$ patients and LDH was elevated in $15(20 \%)$ patients. FDP, D-dimer and IL-6 were elevated in 8 (10.7\%) patients (Table 3). Liver enzymes such as alanine transaminase and aspartate transaminase were deranged in $20(26.67 \%)$ patients. Most patients who required oxygen therapy had elevated levels of CRP, D-dimer, FDP, IL-6, LDH, procalcitonin and serum ferritin than those who did not require it $(P<0.05)$. Dynamic changes in the levels of these inflammatory markers were observed at day 7 after admission (Table 4).

Initially, 3 patients required bilevel positive airway pressure (BiPaP) therapy, but they were placed on mechanical ventilation after they developed ARDS. In the remaining patients who were on oxygen therapy, saturation was maintained with the help of nasal prongs or face masks.

A total of $72(96 \%)$ patients were discharged after they made a complete recovery, and only after they had tested negative for COVID-19 twice over 24 hours (Table 5). Three patients demised after they progressed to ARDS. Mean hospital stay was 9.2 days; for patients who required oxygen therapy, it was 10.2 days. 


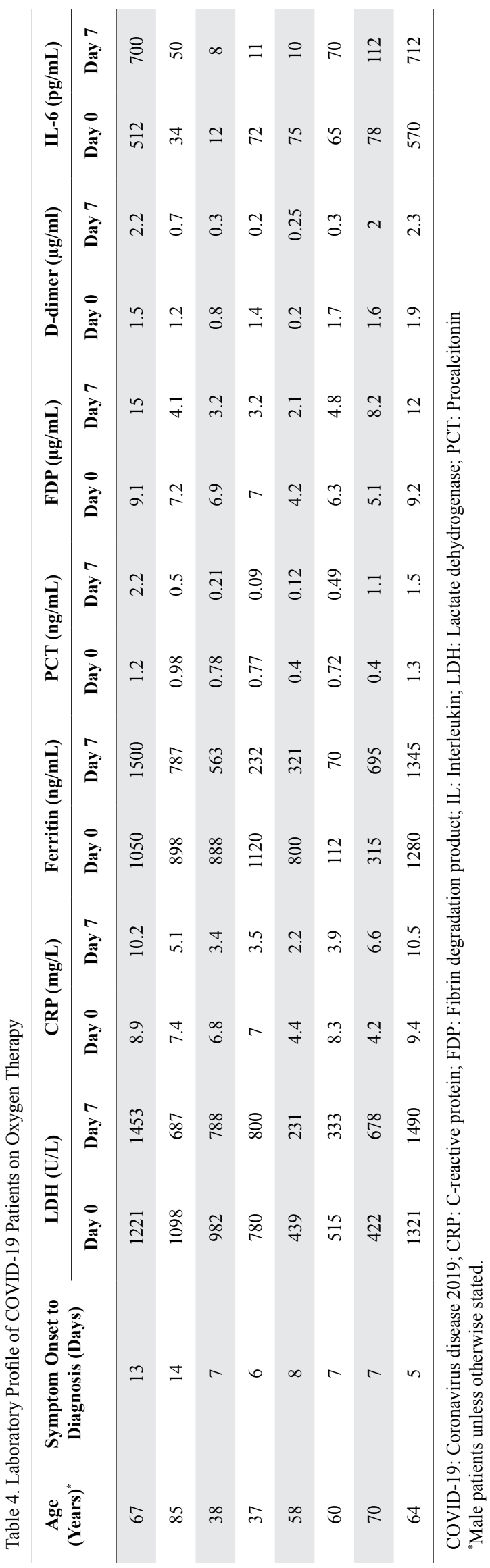

Since no treatment was available for COVID-19, patients were treated according to the guidelines of the Indian Council of Medical Research and were rigorously monitored for any side effects such as QT prolongation in ECG. ${ }^{8}$ Consequently, all patientsasymptomatic or who had mild symptoms-were given hydroxychloroquine $400 \mathrm{mg}$ on day 1 and $200 \mathrm{mg}$ for 4 days. Patients who required oxygen therapy were also given lopinavir/ritonavir, hydroxychloroquine and broad-spectrum antibiotics until culture results were obtained. Gastritis (34.7\%) and diarrhoea $(25.3 \%)$ were the most common side effects reported by patients (Table 5). In 1 patient who developed chest pain, ECG was suggestive of tall $\mathrm{T}$-waves but troponin $\mathrm{T}$ was normal.

\section{Discussion}

In our study, the mean age-38.46 years-of patients was lower than those reported by Wang et $\mathrm{al}^{9}{ }^{9}$ Chen et $\mathrm{al}^{10}$ and Huang et $\mathrm{al}^{11}$ which were $56.0,55.5$ and 49.0 years, respectively. This finding could be attributed to the inclusion of all consecutive cases including patients who were aged $<18$ years old. The higher mean age - 59.8 years - of patients who required oxygen therapy than those-with a mean age of 35.4 years-who were able to maintain saturation at room air suggests that older patients are likely to be at greater risk of lung injury and require oxygen therapy. Since $6(75 \%)$ patients who were on oxygen therapy had underlying comorbidities, this finding suggests that individuals with a history of comorbidities are at increased risk of becoming severely infected by COVID-19 and may develop pneumonia.

The preponderance of male $(74.66 \%)$ patients in our study also corroborated similar findings by Chen et $\mathrm{al}^{10}$ and Huang et $\mathrm{al}^{11}$ who found that $73 \%$ of patients in their studies were men; Wang et al, ${ }^{9}$ however, reported fewer male $(54.3 \%)$ patients in their study. Incidentally, all patients who required oxygen therapy in our study were men. The finding of a male preponderance in our study could be attributed to the observation that traditionally, more men than women travel for educational and work opportunities and more of them would be exposed to COVID-19 and become infected. In another study, $\mathrm{Cai}^{12}$ suggested that the higher prevalence of smoking - which increases expression of angiotensinconverting enzyme 2 (ACE2) receptors in the lungsamong men may make them more vulnerable to 
Table 5. Patient Outcomes and Adverse Events During Hospital Stay $(\mathrm{N}=75)$

\begin{tabular}{|c|c|c|c|c|c|c|c|}
\hline \multirow{2}{*}{ Age (years) } & \multicolumn{2}{|c|}{ Outcome } & \multirow{2}{*}{$\begin{array}{c}\text { Mean Duration of } \\
\text { First Negative Result } \\
\text { (Days) }\end{array}$} & \multirow{2}{*}{$\begin{array}{c}\text { Mean Duration of } \\
\text { Second Negative Result } \\
\text { (Days) }\end{array}$} & \multicolumn{3}{|c|}{ Adverse Events } \\
\hline & $\begin{array}{l}\text { Mean Hospital } \\
\text { Stay (Days) }\end{array}$ & $\begin{array}{l}\text { Discharged } \\
\quad(n=72)\end{array}$ & & & $\begin{array}{c}\text { Gastritis } \\
(n=21)\end{array}$ & $\begin{array}{c}\text { Diarrhoea } \\
(\mathrm{n}=19)\end{array}$ & $\begin{array}{l}\text { Others } \\
(\mathrm{n}=6)\end{array}$ \\
\hline $1-18$ & 9 & 9 & 8 & 9 & 0 & 0 & 0 \\
\hline $19-30$ & 10.75 & 22 & 9.25 & 10.75 & 7 & 5 & 2 \\
\hline $31-45$ & 10.2 & 19 & 8.8 & 10.2 & 6 & 4 & 1 \\
\hline $46-60$ & 7 & 13 & 5.5 & 7 & 5 & 3 & 2 \\
\hline$>61$ & 7.5 & 9 & 6.25 & 7.5 & 8 & 7 & 1 \\
\hline
\end{tabular}

infection by COVID-19. In their study of single-cell sequencing, Zhao et $\mathrm{al}^{13}$ found a predominance of ACE2 expression in Asian men which might explain the higher incidence of COVID-19 in this subgroup than women and men of other ethnicities.

Since a significant number of our patients were asymptomatic $(45.3 \%)$, this finding indicates that a large number of individuals in North India who had been infected by COVID-19 continued to remain asymptomatic. It is therefore important to identify such cases to contain the rapid spread of human-tohuman transmission in COVID-19. ${ }^{14}$ Although $19.51 \%$ of patients in our study had dyspnoea, this finding was lower than the incidence of $24.9 \%$ and $57.6 \%$ reported by meta-analyses of COVID-19 patients in China and Spain, respectively. ${ }^{15,16}$ A study with a larger sample size is needed to validate these findings.

The finding that all patients who required oxygen therapy had demonstrated lymphopaenia in their hemogram could suggest a potential role for lymphopaenia to be used as a prognostic marker in COVID-19. Additionally, the finding that other markers of inflammation such as CRP, D-dimer, FDP, IL-6, serum ferritin and liver enzymes were significantly elevated $(P<0.05)$ in patients who required oxygen therapy than those who did not was similar to that of Liu et $\mathrm{a}^{17}$ who found that these markers were more elevated in severe than non-severe patients. This suggests that besides lung injury, the novel coronavirus may be associated with cytokine release syndrome and liver injury. These findings echoed the observation by Ruan et $\mathrm{al}^{18}$ that inflammatory markers may potentially play a role in predicting mortality in COVID-19 patients in China.
During the H1N1 pandemic in 2009, Paquette et al ${ }^{19}$ reported significant elevation in IL-6 levels in patients who were infected by the virus and required critical care than those who did not require such care. They also reported higher IL-6 levels in patients who had demised $(22.22 \%)$ compared to survivors $(77.77 \%)$. In COVID-19 patients, Liu et a ${ }^{17}$ reported a significant decrease in IL-6 levels and improved CT findings in $81.33 \%$ of patients after they were treated. In our study, IL-6 levels were elevated in all patients who required oxygen therapy and they varied according to disease progression or remission. This observation corroborated the finding of the study by Bhandari et $\mathrm{al}^{20}$ who had emphasised the role that IL-6 levels could play in cytokine release syndrome in a subgroup of COVID-19 patients.

A limitation of our study was that it was a single-centre trial that sampled the population from a specific region in North India. Treatment guidelines and testing protocols were also revised throughout the duration of the study.

\section{Conclusion}

COVID-19 is spreading at an unprecedented rate from around the world and has negatively impacted the global economy and public health. The findings of this study on the clinical and epidemiological profile of early cases of COVID-19 patients suggested that older adults with $\geq 1$ comorbidity had potentially poorer clinical outcomes. A thorough evaluation of inflammatory markers might help in risk stratification of patients who are treated for COVID-19. More studies are needed to understand the full clinical and epidemiological spectrum of this disease. 


\section{REFERENCES}

1. Zhu N, Zhang D, Wang W, Li X, Yang B, Song J, et al. A novel coronavirus from patients with pneumonia in China, 2019. N Engl J Med 2020;382:727-33.

2. Walls AC, Park YJ, Tortorici MA, Wall A, McGuire AT, Veesler D. Structure, function and antigenicity of the SARS-CoV-2 spike glycoprotein. Cell 2020;181:281-92.

3. Cucinotta D, Vanelli M. WHO declares COVID-19 a pandemic. Acta Biomed 2020;91:157-60.

4. Goh KJ, Choong MC, Cheong EH, Kalimuddin S, Duu Wen S, Phua GC, et al. Rapid progression to acute respiratory distress: review of current understanding of critical illness from COVID-19 infection. Ann Acad Med Singapore2020;49:108-18

5. Koh D, Cunningham AC. Counting coronavirus disease 2019 (COVID-19) cases: case definitions, screened populations and testing techniques matter. Ann Acad Med Singapore 2020;49:161-5.

6. Mehta P, McAuley DF, Brown M, Sanchez E, Tattersall RS, Manson JS, et al. COVID-19: consider cytokine storm syndromes and immunosuppression. Lancet 2020;395:1033-4.

7. Ministry of Health and Family Welfare, India. COVID-19 Statewise Status. Available at: www.mohfw.gov.in. Accessed on 11 April 2020

8. Bhatnagar T, Murhekar MV, Soneja M, Gupta N, Giri S, Wig $\mathrm{N}$, et al. Lopinavir/ritonavir combination therapy amongst symptomatic coronavirus disease 2019 patients in India: protocol for restricted public health emergency use. Indian $\mathrm{J}$ Med Res 2020;151:184-9.

9. Wang D, Hu B, Hu C, Zhu F, Liu X, Zhang J et al. Clinical characteristics of 138 hospitalized patients with 2019 novel coronavirus-infected pneumonia in Wuhan, China. JAMA 2020;323:1061-9.

10. Chen N, Zhou M, Dong X, Qu J, Gong F, Han Y, et al. Epidemiological and clinical characteristics of 99 cases of 2019 novel coronavirus pneumonia in Wuhan, China: a descriptive study. Lancet 2020;395:507-13.

11. Huang C, Wang Y, Li X, Ren L, Zhao J, Hu Y, et al. Clinical features of patients infected with 2019 novel coronavirus in Wuhan, China. Lancet 2020;395:497-506.

12. Cai H. Sex difference and smoking predisposition in patients with COVID-19. Lancet Respir Med 2020;8:e20.

13. Zhao Y, Zhao Z, Wang Y, Zhou Y, Ma Y, Zuo W. Single-cell RNA expression profiling of ACE2, the receptor of SARS-CoV-2. Am J Respir Crit Care Med 2020;doi:10.1164/rccm.202001-0179LE.

14. Li Q, Guan X, Wu P, Wang X, Zhou L, Tong Y, et al. Early transmission dynamics in Wuhan, China, of novel coronavirusinfected pneumonia. N Engl J Med 2020;382:1199-207 .

15. Fu L, Wang B, Yuan T, Chen X, Ao Y, Fitzpatrick T, et al. Clinical characteristics of coronavirus disease 2019 (COVID-19) in China: a systematic review and meta-analysis. J Infect 2020;80:656-65.

16. Rojo JMC, Santos JMA, Núñez-Cortés JM, Bermejo CL, Rincón JMR, Roy-Vallejo E, et al. Clinical characteristics of patients hospitalized with COVID-19 in Spain: results from the SEMI-COVID-19 Network. 2020. MedRxiv 2020;doi:https://doi.org/ 10.1101/2020.05.24.20111971.

17. Liu T, Zhang J, Yang Y, Ma H, Li Z, Zhang J, et al. The potential role of IL-6 in monitoring severe case of coronavirus disease 2019. MedRxiv 2020;doi:https://doi.org/10.1101/2020.03.01.20029769.

18. Ruan Q, Yang K, Wang W, Jiang L, Song J. Clinical predictors of mortality due to COVID-19 based on an analysis of data of 150 patients from Wuhan, China. Intensive Care Med 2020;46:846-8.

19. Paquette SG, Banner D, Zhao Z, Fang Y, Huang SSH, León AJ, et al. Interleukin- 6 is a potential biomarker for severe pandemic H1N1 influenza A infection. PLoS One 2012;7:e38214.

20. Bhandari S, Sharma S, Bhargava A, Keswani P, Sharma R, Shekhawat A. Inflammatory markers in COVID-19. Ann Acad Med Singapore 2020;49:393-7. 